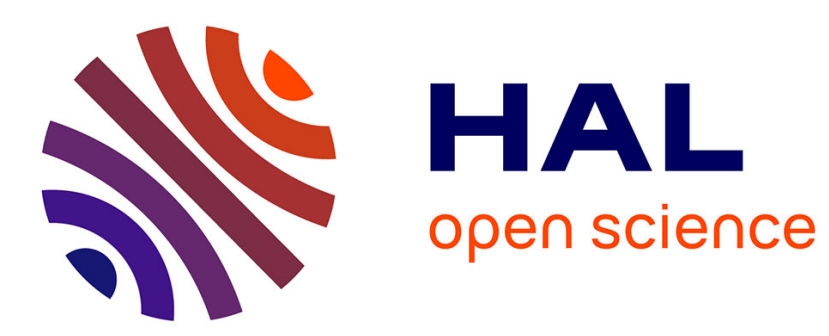

\title{
Optimal sound absorbing and manufacturable two-dimensional, hexagonal-like porous structure
}

Camille Perrot, F. Chevillotte, R. Panneton

\section{To cite this version:}

Camille Perrot, F. Chevillotte, R. Panneton. Optimal sound absorbing and manufacturable twodimensional, hexagonal-like porous structure. Session 1pPAa: Physical Acoustics, Jun 2008, Paris, France. pp.045004, 10.1121/1.2972138 . hal-00816946

\section{HAL Id: hal-00816946 https://hal.science/hal-00816946}

Submitted on 23 Apr 2013

HAL is a multi-disciplinary open access archive for the deposit and dissemination of scientific research documents, whether they are published or not. The documents may come from teaching and research institutions in France or abroad, or from public or private research centers.
L'archive ouverte pluridisciplinaire HAL, est destinée au dépôt et à la diffusion de documents scientifiques de niveau recherche, publiés ou non, émanant des établissements d'enseignement et de recherche français ou étrangers, des laboratoires publics ou privés. 


\title{
Proceedings of Meetings on Acoustics
}

\author{
155th Meeting \\ Acoustical Society of America \\ Paris, France \\ 29 June - 4 July 2008 \\ Session 1pPAa: Physical Acoustics
}

\section{1pPAa11. Optimal sound absorbing and manufacturable two-dimensional, hexagonal-like porous structure}

\author{
Camille Perrot*, Fabien Chevillotte and Raymond Panneton \\ *Corresponding author's address: Mechanical Engineering, Université de Sherbrooke, 2500, Boul. de l'Université, \\ Sherbrooke, J1K-2R1, Québec, Canada, camille.perrot@usherbrooke.ca
}

Results from a numerical study examining micro-macro relations linking local geometry parameters to sound absorption properties are presented. For an hexagonal structure of solid fibers, the porosity Phi, the thermal characteristic length $\Lambda^{\prime}$, the static viscous permeability k0, the tortuosity $\alpha \infty$, the viscous characteristic length $\Lambda$, and the sound absorption coefficient are computed. Numerical solutions of the steady Stokes and electrical equations are employed to provide k0, $\alpha \infty$, and $\Lambda$. Hybrid estimates based on direct numerical evaluation of Phi, $\Lambda^{\prime}, \mathrm{k} 0, \alpha \infty, \Lambda$ and the analytical model derived by Johnson, Allard, and Champoux are used to relate varying (i) throat size, (ii) pore size, and (iii) fibers cross-section shapes to the sound absorption spectrum. The result of this paper tends to demonstrate the important effect of throat size in the sound absorption level, cell size in the sound absorption frequency selectivity, fibers cross-section shape in the porous material weight reduction. In a hexagonal porous structure with solid fibers, the sound absorption level will tend to be maximized with a $48+/-10$ micrometers throat size corresponding to an intermediate resistivity, a $13+/-8$ micrometers fiber radius associated with relatively small inter-fiber distances, and convex triangular cross-section shape fibers allowing weight reduction.

Published by the Acoustical Society of America through the American Institute of Physics 


\title{
Optimal sound absorbing and manufacturable two-dimensional, hexagonal-like porous structure
}

\author{
Camille Perrot, Fabien Chevillotte, and Raymond Panneton \\ Groupe d'Acoustique de l'Université de Sherbrooke (GAUS), Department of mechanical engineering, \\ Université de Sherbrooke, Canada, (Qc) J1K 2RI \\ camille.perrot@usherbrooke.ca fabien.chevillotte@usherbrooke.ca raymond.panneton@usherbrooke.ca
}

\begin{abstract}
Results from a numerical study examining micro-macro relations linking local geometry parameters to sound absorption properties are presented. For an hexagonal structure of solid fibers, the porosity $\Phi$, the thermal characteristic length $\Lambda^{\prime}$, the static viscous permeability $k_{0}$, the tortuosity $\alpha_{\infty}$, the viscous characteristic length $\Lambda$, and the sound absorption coefficient are computed. Numerical solutions of the steady Stokes and electrical equations are employed to provide $k_{0}, \alpha_{\infty}$, and $\Lambda$. Hybrid estimates based on direct numerical evaluation of $\Phi, \Lambda^{\prime}, k_{0}, \alpha_{\infty}$, $\Lambda$ and the analytical model derived by Johnson, Allard, and Champoux are used to relate varying (i) throat size, (ii) pore size, and (iii) fibers cross-section shapes to the sound absorption spectrum. The result of this paper tends to demonstrate the important effect of throat size in the sound absorption level, cell size in the sound absorption frequency selectivity, fibers cross-section shape in the porous material weight reduction. In a hexagonal porous structure with solid fibers, the sound absorption level will tend to be maximized with a $48 \pm 10 \mu \mathrm{m}$ throat size corresponding to an intermediate resistivity, a $13 \pm 8 \mu \mathrm{m}$ fiber radius associated with relatively small inter-fiber distances, and convex triangular cross-section shape fibers allowing weight reduction.
\end{abstract}




\section{Introduction}

A major issue in automobile, aeronautical, and building industries concerns the need to increase or adapt the sound absorption spectrum of commonly used sound absorbing materials. However, the most advanced models used to characterize and predict sound absorbing material performances are mainly based on interdependent macroscopic parameters which do not take explicitly into account the local geometry of the porous media (i.e., its microstructure). For these reasons, optimizing sound absorbing materials from their fabrication remains a difficult task mostly done by trial and error. A strict optimization method would firstly rely on our ability to predict the acoustic properties of porous media from the description of their local geometry. Secondly, it would propose pertinent realistic modifications of their microstructure having predictable impacts on their absorption spectrum. The intent of this paper is to present such an optimization procedure following the bottom-up approach (i.e., from an optimized microstructure to the desired acoustical macroscopic behavior).

Starting from micro-physical foundations, the aim of this paper is to illustrate the potential of such a bottom-up approach for microstructure optimization of highly porous open-cell foams and fibrous sound absorbing materials. In the framework of this paper, only the dynamic viscous boundary value problem is considered. The porous structure is a hexagonal lattice of solid fibers in air. For this simple geometry, it is shown how local geometry parameters are related to the sound absorption spectrum of the porous media through the main macroscopic parameters, giving a physical insight for why it was achieved. In particular, we will examine the influence of (i) the throat size, (ii) the cell size, and (iii) the cross-section shape of the fibers (i.e., circle, convex, straight, and concave triangles). An outline of this paper is as follows: In Sec. 2, the model geometry and computational method behind the bottom-up approach is introduced. In Sec. 3, results on a hexagonal structure of solid fibers are presented and discussed. Sec. 4. concludes this talk. 


\section{Numerical calculations}

\subsection{Model geometry}

A typical two-dimensional hexagonal arrangement of fibers having $l$ and $r$ as local characteristic dimensions is depicted in Fig. 1. The fibers (the solid phase) are assumed motionless and diluted in air (the fluid phase). Their cross-sections form the nodes of the hexagons. In this illustration, the crosssections of the fibers are circular. This is a typical case for a porosity $\Phi \approx 0.85$ (or less). There are experimental evidences [1] that the cross-section shape of a foam ligament is evolving from a circle $(\Phi \approx$ $85 \%)$ for low porosity foams to convex $(\Phi \approx 90 \%)$, straight $(\Phi \approx 94 \%)$, and concave $(\Phi \approx 98 \%)$ triangles for high porosity foams. For this reason, the shape of the cross-section will be also considered as a local geometry parameter. See Fig. 2 for an illustration of the different cross-section shapes used in this study.

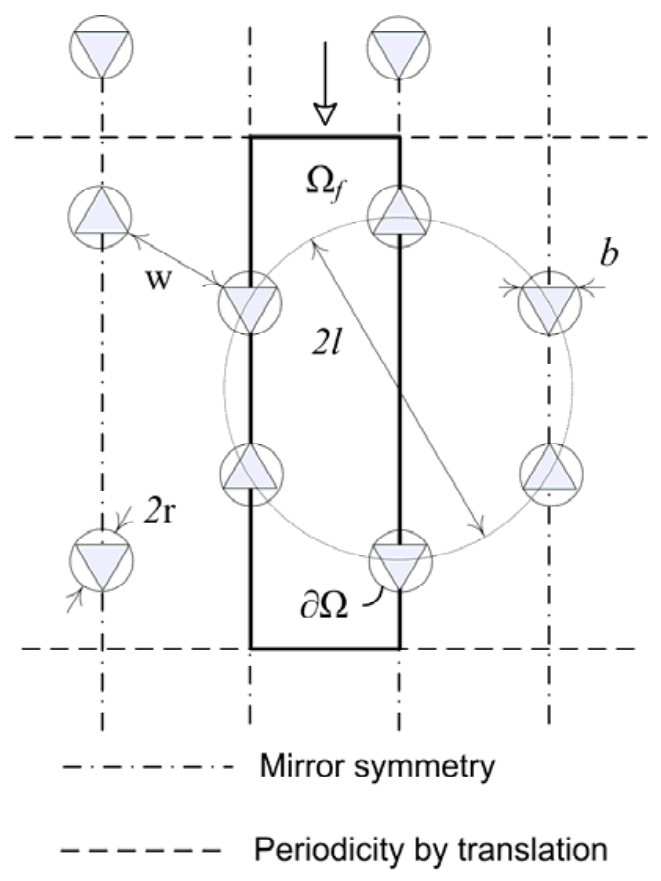

Figure 1. Local geometry model showing solid fibers having circular or triangular cross-section shapes arranged in a hexagonal pattern. Triangular cross-sections are considered to be inscribed in the corresponding circular ones. Numerical computations are performed at the scale of a vertical identified Periodic Unit Cells (PUC). 


\section{Cross-section shape}

Porosity (\%)

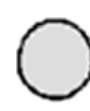

86.2

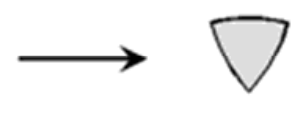

90.9

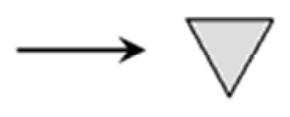

92.6

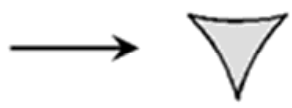

94.6

Figure 2. Cross-section shapes and associated porosity values considered in this study. The porosity values are obtained with $\mathrm{r}=32 \mu \mathrm{m}$ and $\mathrm{w}=70 \mu \mathrm{m}$. Triangular cross-section shapes (convex, straight, concave) are inscribed in the initial circular cross-section.

\subsection{Computational method}

The problem is addressed in three main steps: 1) solve numerically the asymptotic low (steady Stokes) and high (electric) frequency viscous boundary value problems using the finite element method [2]; 2) compute the static viscous permeability $k_{0}$, the viscous characteristic length $\Lambda$, and the tortuosity $\alpha_{\infty}$, as defined by Johnson et al. [3] by appropriate volume averaging of the corresponding asymptotic velocity fields; 3) derive the frequency-dependent viscous and thermal response functions such as the effective density $\rho(\omega)$ and bulk modulus $K(\omega)$ of the entrained fluid in the rigid frame porous medium from the previously computed macroscopic parameters using Johnson et al. [3] and Champoux and Allard [4, 5] analytical models, respectively, from which an approximate but robust description of propagation and absorption phenomena of an acoustic wave through a porous medium is entirely known [5]. Then, modification of the local geometry parameters by an iteration process allows to maximize the area under the sound absorption coefficient curve in the frequency range of interest (here, from 10 to $10000 \mathrm{~Hz}$ ).

\section{Results and discussion}

A number of local geometry calculations are presented to illustrate the use of the computational method in maximizing the absorption coefficient of a hard backed two-dimensional hexagonal porous structure having a total thickness of $25 \mathrm{~mm}$ for varying (i) throat sizes, (ii) cell sizes, and (iii) fiber cross-section shapes. To do so, the following sound absorption performance index will be used:

$$
I=\frac{1}{N} \sum_{i=1}^{N} \alpha\left(\omega_{i}\right)
$$


where $\alpha\left(\omega_{i}\right)$ is the normal incidence sound absorption computed at the $\mathrm{i}^{\text {th }}$ angular frequency, and $N$ is the number of discrete angular frequencies used in the frequency range of interest - here 10 to $10000 \mathrm{~Hz}$. For a given configuration, this indicator has to be maximized to yield the optimized local parameters.

\subsection{Multi-variable analysis}

A multi-variable optimization was performed for different values of the throat size w and the fiber radius $\mathrm{r}$ in the range $w=[20,200] \mu \mathrm{m}$ and $r=[1,200] \mu \mathrm{m}$, with $\mathrm{c}$ being assigned to circular and triangular cross-section shapes. The results are shown in Fig. 3. For both cross-section shapes, the resulting response surfaces shown by Fig. 3 (top) seem to demonstrate the existence of global maximums. For the case of the circular cross-section shape, $I_{o p t}=85.47 \%$ at $(w, r)_{\text {opt }}=(48,6) \mu \mathrm{m}$. For the case of the triangular cross-section shape, $I_{\text {opt }}=85.91 \%$ at $(w, r)_{\text {opt }}=(48,13) \mu \mathrm{m}$. Furthermore, it appears clearly in Fig. 3 (bottom) that, contrary to the triangular cross-section case for which the optimal throat size is constant for varying radius (straight dotted line), the circular cross-section case shows a more complex relation between optimal throat size and radius (curved dotted line). This different behavior might be interpreted geometrically as follows. Given two triangular cross-sections, a fiber radius increase is not significantly modifying the solid surface seen by the acoustic wave in the vicinity of the throat. On the contrary, for circular cross-sections, a fiber radius increase is associated with a non-neglectable solid surface increase which is seen by the acoustic wave in the vicinity of the throat, except if the throat size is also increased. Finally, it is of practical interest to mention that, if the fabrication process is advanced enough to produce a fiber pattern keeping the optimal throat size, a relative large tolerance [5 $\mu \mathrm{m}-150$ $\mu \mathrm{m}]$ is acceptable in terms of fiber radius to stay with $95 \%$ of $I_{o p t}$. 

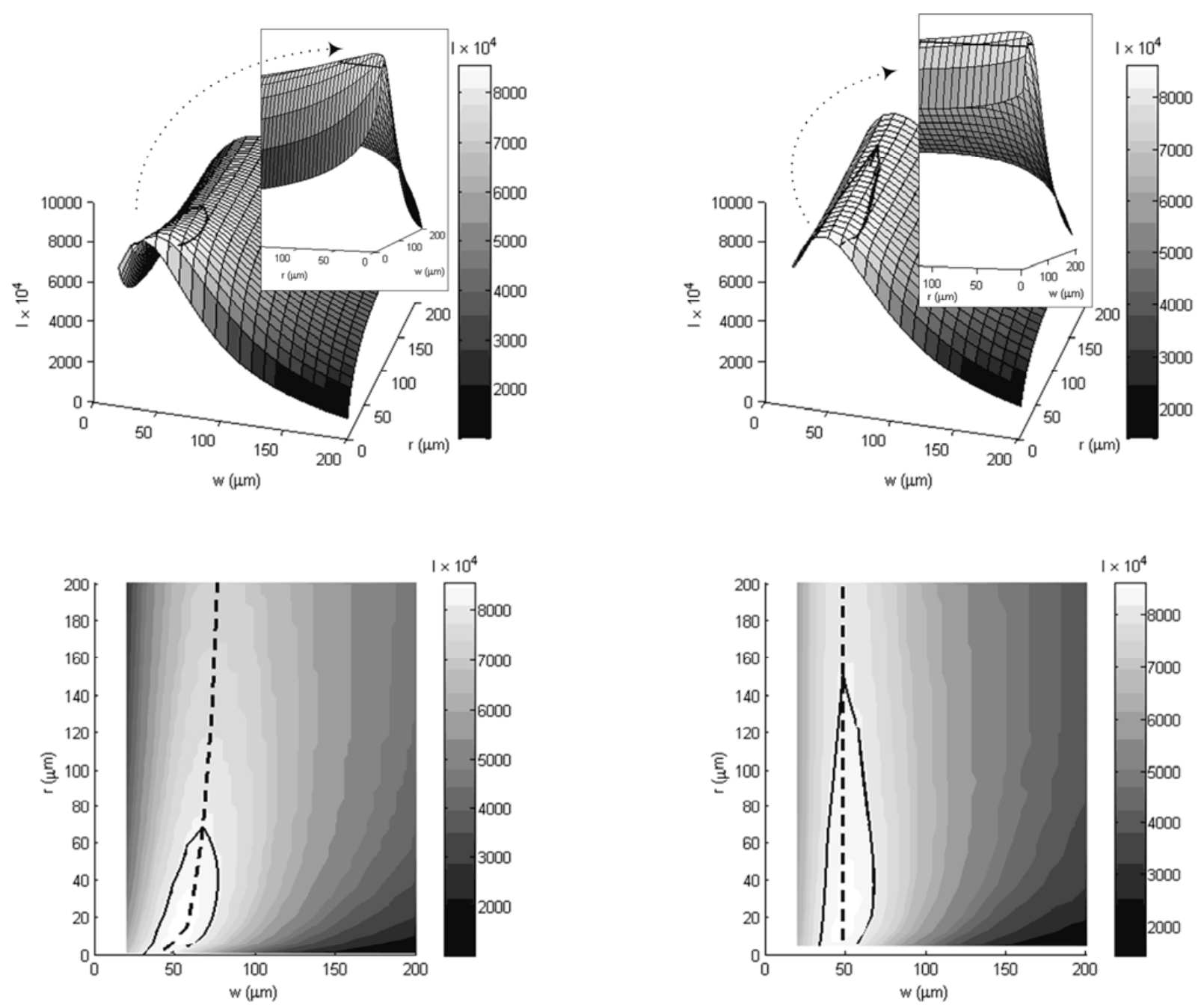

Figure 3. Response surfaces (top) for the circular (left) and triangular (right) cross-section shape cases and associated 2D map (bottom). Straight lines are delimiting the $95 \%$ Iopt zone, and dotted lines are associated with the optimal $(\mathrm{w}, \mathrm{r})$ couples.

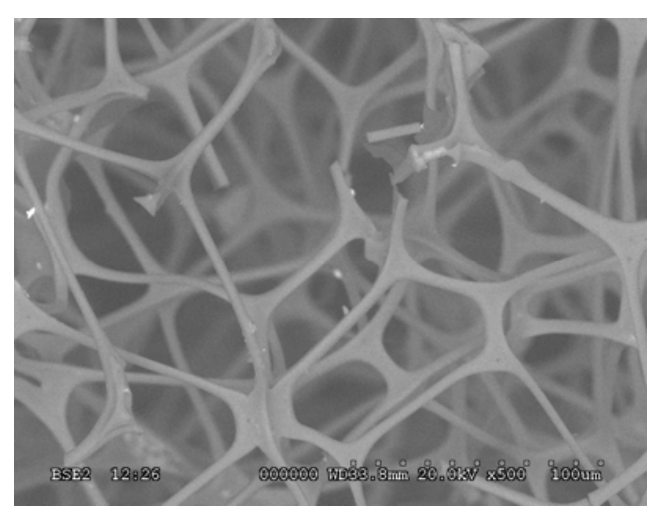

Figure 4. Scanning electron micrograph of a real efficient sound absorbing melamine foam sample. 


\subsection{Comparison with experimental data}

Finally, it would be interesting, however not necessary, to compare the results of this bottom-up approach to experimental data. The melamine foam is known to be one of the best acoustic materials in terms of sound absorption. It is often used to design anechoic chambers, and in aeronautic applications. It possesses a highly porous open-cell structure, with very elongated thin ligaments. In some extent, it could be seen as a fibrous structure such as the one described in Fig. 1. The cross-section shape of a melamine foam ligament is made of concave triangles. A scanning electron micrograph of a real melamine foam sample is presented in Fig. 4. A rough estimate of the local geometry parameters can be obtained from such micrographies, yielding [ $4.3 \pm 0.3 \mu \mathrm{m}, 46.5 \pm 31.3 \mu \mathrm{m}]$ (the average length of a ligament on a micrograph is taken as the average length between two ligaments in the model geometry) and $[2.5 \pm 0.2 \mu \mathrm{m}, 41.5 \pm 31.7 \mu \mathrm{m}]$. By reporting this couple of local geometry values in the chart of Fig. 3 , one can see that this local geometry configuration is lying in the best absorption region.

\subsection{Limitations and future works}

For practical trend exploration of sound absorption due to porous media morphology, simplifications were made and refined analytical models were not used. However, in the limiting case of fiber radius tending to zero, porosity is tending to one (i.e., very small fibers concentration) which means that the dimensionless shape factors must tend to zero and that the validity of the results obtained with JohnsonAllard-Champoux model become questionable. For this reason, a computational check of was carried out using the refined analytical models in the optimal geometric configuration associated with fibers of circular cross-section shape. The differences for $I$ between Johnson-Allard-Champoux and the refined models were found to be less than $3 \%$ (refined models are predicting better sound absorption performances - notably in the low frequency range). Future works should include (i) a weighting of the low frequency range in the computation of a sound absorption performance index, as well as (ii) a close examination of the acoustical macro-behavior in the limit of small radius using refined models. In particular, the notion of an optimum radius appearing while using the Johnson-Allard-Champoux model 
needs to be checked. This would also require significant modifications of the fluid-solid boundary conditions.

\section{Concluding remarks}

The results of our bottom-up approach for microstructure optimization of sound absorbing materials are summarized in this section. For a given fiber radius, an optimal throat size controlling the sound absorption level can be found, corresponding to an intermediate static airflow resistivity. By contrast, given an optimal throat size, the fiber radius (i.e. cell size) is essentially modulating the absorption curve. It is worth mentioning that the optimal absorption curve is the one which is minimizing the viscous characteristic length at constant throat size. This property can be used as a design guide for sound absorption optimization. Another observation is the fact that for a given thermal characteristic length, as the porosity increases from its circular cross-section value to its concave triangular cross section value, the throat size reduces with the viscous characteristic length, enhancing only slightly the sound absorption coefficient of the porous structure but increasing notably its porosity (i.e., reduction of bulk density or weight). Finally, practical investigation charts have been proposed to indicate local geometry parameters tending to maximize the sound absorption coefficient. The validity of these charts was corroborated by comparison with the measured local geometry parameters of a real efficient sound absorbing melamine foam. This confirms the potential of such a bottom-up approach for microstructure optimization of sound absorbing materials. 


\section{References}

[1] A. Bhattacharya, V. V. Calmidi and R. L. Mahajan, "Thermophysical properties of high porosity metal foams, ” Int. J. Heat Mass Transfer 45, 1017 (2002).

[2] C. Perrot, F. Chevillotte, and R. Panneton, "Dynamic viscous permeability of an open-cell aluminum foam: Computations versus experiments,” J. Appl. Phys. 103, 024909 (2008).

[3] D. L. Johnson, J. Koplik, and R. Dashen, "Theory of dynamic permeability and tortuosity in fluidsaturated porous media, ” J. Fluid Mech. 176, 379-402 (1987).

[4] Y. Champoux and J. F. Allard, "Dynamic tortuosity and bulk modulus in air-saturated porous media, ” J. Appl. Phys. 70, 1975-1979 (1991).

[5] J. F. Allard, Propagation of sound in porous media, "Modelling sound absorbing materials," edited by Elsevier Applied Science, (Elesevier Science Publishers LTD, New York and London, 1993), pp. 284. 